\title{
Reactor neutrino experiments
}

Y. WANG $(*)$

Institute of High Energy Physics - Yu-Quan Road 19B, Beijing, 100049, P.R. China

Summary. - Reactor neutrinos played a very important role in the history of neutrino studies. In addition to the neutrino discovery and the precision measurement of neutrino oscillation parameters of $\theta_{12}$ and $\Delta m_{21}^{2}$, there is a major achievement very recently by the Daya Bay, RENO and Double Chooz experiments. They discovered the non-zero neutrino mixing angle, $\theta_{13}$, hence completed the picture of neutrino oscillations. The next generation reactor neutrino experiments will be able to determine the neutrino mass hierarchy and improve significantly the precision of $\theta_{12}, \Delta m_{21}^{2}$ and $\Delta m_{31}^{2}$. Reactor neutrino experiments can be also used to study sterile neutrinos.

\section{1. - Introduction}

Oscillation is a fundamental property of neutrinos, which is related to the neutrino masses and mixing, and may generate leptonic CP violation to explain the matterantimatter asymmetry of the Universe. For two-flavor oscillation in vacuum, the transition oscillation probability from $\nu_{\alpha}$ to $\nu_{\beta}$ is expressed as

$$
P\left(\nu_{\alpha} \rightarrow \nu_{\beta}\right)=\sin ^{2} 2 \theta \sin ^{2}\left(1.27 \frac{\Delta m^{2}}{\mathrm{eV}^{2}} \cdot \frac{L}{\mathrm{~m}} \cdot \frac{\mathrm{MeV}}{E}\right)
$$

where $\sin ^{2} 2 \theta$ denotes the oscillation amplitude and $\Delta m^{2} L / E$ represents the oscillation frequency. For three generations of neutrinos, the oscillation is often described by the $3 \times 3$ neutrino mixing matrix,

$$
\left(\begin{array}{c}
\nu_{e} \\
\nu_{\mu} \\
\nu_{\tau}
\end{array}\right)=\left(\begin{array}{ccc}
V_{e 1} & V_{e 2} & V_{e 3} \\
V_{\mu 1} & V_{\mu 2} & V_{\mu 3} \\
V_{\tau 1} & V_{\tau 2} & V_{\tau 3}
\end{array}\right)\left(\begin{array}{c}
\nu_{1} \\
\nu_{2} \\
\nu_{3}
\end{array}\right)
$$

$\left(^{*}\right)$ E-mail: yfwang@ihep.ac.cn

(c) Società Italiana di Fisica 
Here the so-called Pontecorvo-Maki-Nakagawa-Sakata (PMNS) matrix $V$ can be written as $[1,2]$,

$$
V=\left(\begin{array}{ccc}
1 & 0 & 0 \\
0 & c_{23} & s_{23} \\
0 & -s_{23} & c_{23}
\end{array}\right) \cdot\left(\begin{array}{ccc}
c_{13} & 0 & s_{13} \\
0 & e^{-i \delta} & 0 \\
-s_{13} & 0 & c_{13}
\end{array}\right) \cdot\left(\begin{array}{ccc}
c_{12} & s_{12} & 0 \\
-s_{12} & c_{12} & 0 \\
0 & 0 & 1
\end{array}\right)
$$

where $c_{i j}=\cos \theta_{i j}$ and $s_{i j}=\sin \theta_{i j}$ for $(i, j=1,2,3)$.

There are six independent parameters in the neutrino oscillation. $\theta_{12}, \Delta m_{21}^{2}, \theta_{23}$ and $\left|\Delta m_{32}^{2}\right|$ have been known for more than 10 years, $\theta_{13}$ has been recently measured, while the sign of $\Delta m_{32}^{2}$, often referred to as neutrino mass hierarchy, and the CP phase $\delta$ are unknown. In this talk, I will review the progress of reactor neutrino experiments for improved measurements on $\theta_{12}$ and $\Delta m_{21}^{2}$, and the discovery of non-zero $\theta_{13}$. Future prospects will be given, particularly for the neutrino mass hierarchy. Experiments to study sterile neutrinos using reactor neutrinos are also introduced.

\section{2. - Reactor neutrinos and past experiments}

It is well known that nuclear fission reactions can produce abundant neutrinos. In fact, a typical reaction can produce on average 6 neutrons which then decay into 6 neutrinos. A typical $3 \mathrm{GW}$ (thermal) nuclear power reactor can generate $6 \times 10^{20}$ electron antineutrinos per second. The energy spectrum of reactor neutrinos follows approximately an exponential power law, as shown in the left panel of Figure 1 [3].

Reactor neutrinos are detected by the liquid scintillator with a chemical formula of $C_{n} H_{2 n+1}$ which contains abundant hydrogen atoms. Through the reaction of $\bar{\nu}_{e}+p \rightarrow$ $e^{+}+n$, neutrinos are detected by a coincidence of prompt signal from $e^{+}$via ionization, and a delayed signal from the neutron capture on hydrogen. The cross section of this reaction is proportional to $E_{e} p_{e}$, where $E_{e}$ and $p_{e}$ are the energy and momentum of the positron, respectively. The final detected neutrino energy is approximately the kinetic energy of the positron, plus a constant mass term $(1.8 \mathrm{MeV})$, with a minor correction (10-40 keV) from the neutron kinetic energy. The spectrum is peaked at about $4 \mathrm{MeV}$, as shown in the left panel of Figure 1 [3].

Reactor neutrinos are used for the first time to detect directly neutrinos in 1950's, on the suggestion of Bruno Pontecorvo [4]. It is appropriate for us to celebrate his centennial anniversary by talking about the progress of reactor neutrinos. It is remarkable that the main technique of reactor neutrino experiments has not been changed since then, thanks to the simple technology (coincidence) for background reduction and good scalability (easy and cheap for a large quantity of the liquid scintillator).

Since 1970's, Reactor neutrinos have been used for oscillation studies [3], as shown in the right panel of Figure 1. By looking at the observed number of neutrinos compared to that of expected, the oscillation amplitude can be determined and null results have been reported up to the end of 1990's.

In 2002, KamLAND discovered the reactor neutrino oscillation [5]. This result for the first time unambiguously determined the neutrino mixing angle $\theta_{12}$ and $\Delta m_{21}^{2}$, and was the first observation of the neutrino oscillation using man-made neutrino sources. Together with other experimental results [6-13], neutrino oscillation is thus firmly established. The KamLAND experiment continued to improve their measurement on $\theta_{12}$ and $\Delta m_{21}^{2}$, and recently they reported their latest results by taking the unique opportunity to measure precisely the backgrounds when all the nuclear reactors in Japan were turned 

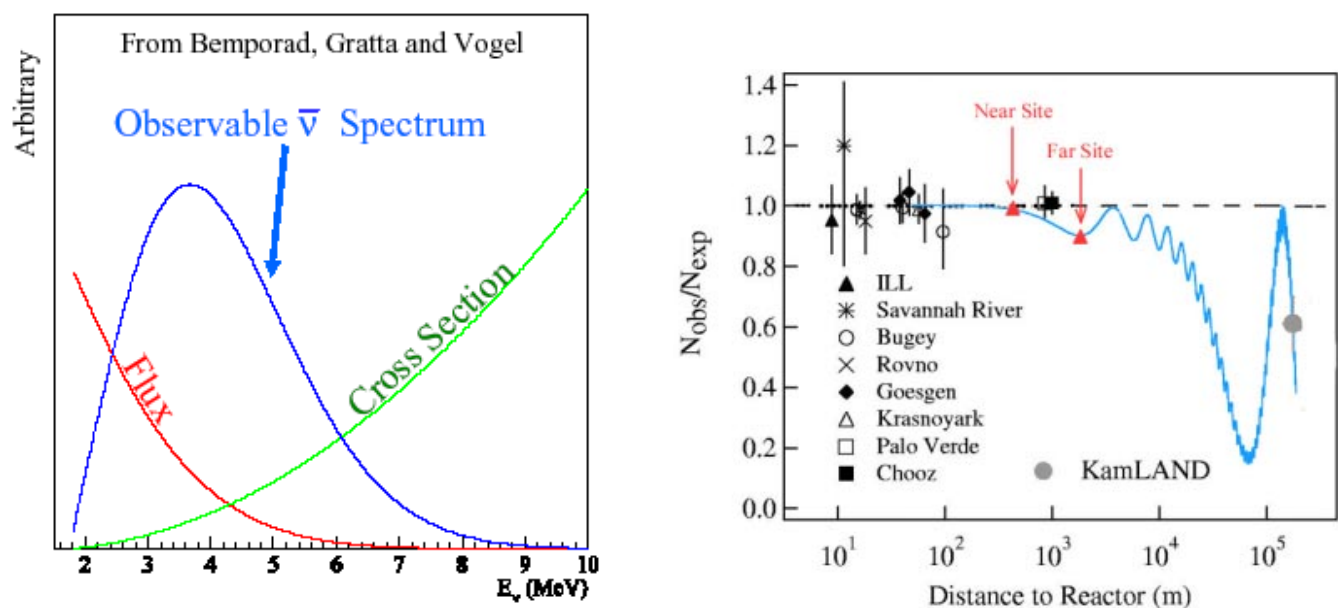

Fig. 1. - Neutrino flux from the reactor, the neutrino cross section and the detected energy spectrum [3] (left panel); Reactor neutrino experiments looking for neutrino oscillations since 1970's (right panel).

off due to the earthquake accident [14]. It is interesting to see that the measured neutrino rate is following the variation of the reactor power and the fitted results are shown in Figure 2.

Reactor neutrinos can also be used for other searches, such as neutrino magnetic moments [15], sterile neutrinos [16], etc.

\section{3. - Current experiments: $\theta_{13}$}

Very recently, the Daya Bay experiment revealed the last neutrino mixing angle $\theta_{13}[17]$, and it was soon confirmed by other experiments $[18,19]$. In fact, reactor neutrino experiments for $\theta_{13}$ started in 2003 with about 8 proposals. In the end, only three experiments, Daya Bay (DYB), Double Chooz (DC), and RENO experiments were approved and performed. Table I lists their main parameters and expected sensitivities.

All the three experiments have a similar detector design. By using identical detectors at the near and far sites, systematic uncertainties are greatly reduced. At each site, as shown in the left panel of Figure 3, neutrino detector modules are sub-merged in a water pool to reduce backgrounds and veto cosmic-rays.

Each neutrino detector module consists of three nested vessels: the most inner one, made of acrylic, is to contain Gd-loaded liquid scintillator; the middle one, also made of acrylic, is for normal liquid scintillator; the outmost one, made of stainless steel, is to contain mineral oil. The right panel of Figure 3 shows the detector module of the Daya Bay experiment as an example.

The exact dimension and the target mass of the detector module in each experiment are different, as listed in Table II. It can be seen clearly that the Daya Bay experiment has the least number of photomultiplier tubes (PMTs), thanks to the reflectors mounted at the top and bottom of the detector modules to save costs. The larger target mass fraction for Daya Bay is achieved due to the thinner oil buffer layer, which has negligible effect on the backgrounds. 


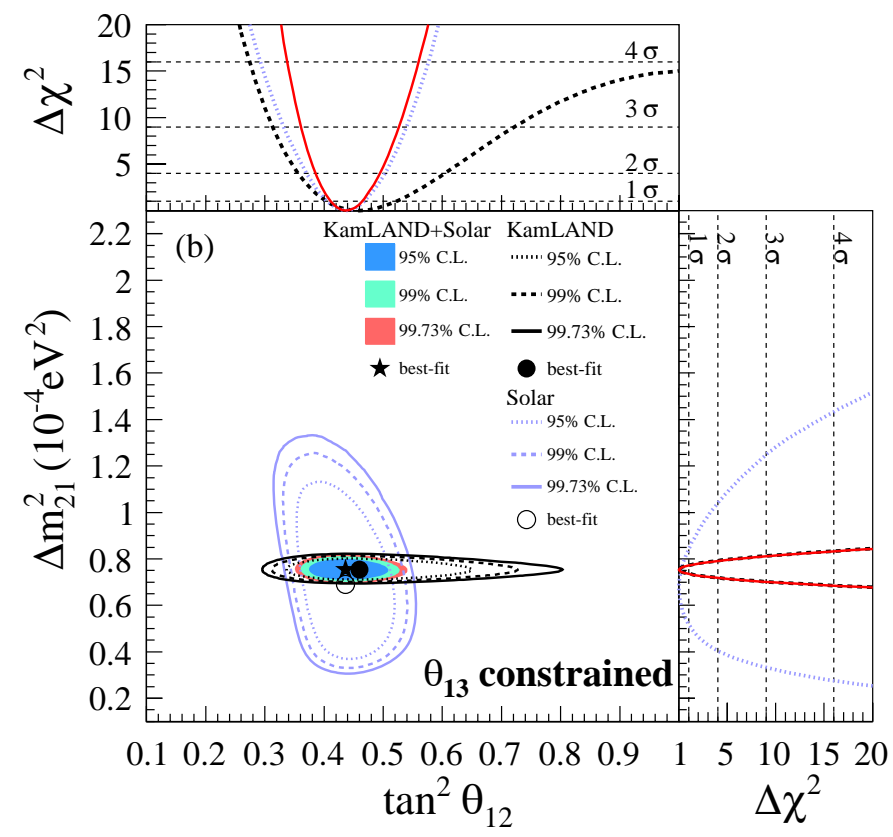

Fig. 2. - The allowed region of $\theta_{12}$ and $\Delta m_{21}^{2}$ from KamLAND and other solar neutrino experiments [14].

TABLE I. - Main parameters of current reactor neutrino experiments and their sensitivities to $\sin ^{2} 2 \theta_{13}$.

\begin{tabular}{|c|c|c|c|c|c|}
\hline Exps. & $\begin{array}{c}\text { Power } \\
(\mathrm{GW})\end{array}$ & $\begin{array}{c}\text { Baseline } \\
\text { (meters) } \\
\text { Near/Far }\end{array}$ & $\begin{array}{c}\text { Detector } \\
\text { (tons) } \\
\text { Near/Far }\end{array}$ & $\begin{array}{c}\text { Overburden } \\
\text { (MWE) } \\
\text { Near/Far }\end{array}$ & $\begin{array}{c}\text { Designed } \\
\text { Sensitivity } \\
\text { (90\% CL) }\end{array}$ \\
\hline DYB & 17.4 & $470 / 576 / 1650$ & $40 / 40 / 80$ & $250 / 265 / 860$ & 0.008 \\
\hline DC & 8.5 & $400 / 1050$ & $8.2 / 8.2$ & $120 / 300$ & 0.03 \\
\hline RENO & 16.5 & $409 / 1444$ & $16 / 16$ & $120 / 450$ & 0.02 \\
\hline
\end{tabular}

TABLE II. - Some of the key parameters of detector modules for the three reactor neutrino experiments.

\begin{tabular}{|c|c|c|c|c|c|c|}
\hline & PMTs & Coverage & P.E. yield & $\begin{array}{c}\text { P.E. yield } \\
\text { /Coverage }\end{array}$ & $\begin{array}{c}\text { target } \\
\text { mass (t) }\end{array}$ & $\begin{array}{c}\text { Target mass } \\
\text { fraction }\end{array}$ \\
\hline DYB & $1928^{\prime \prime}$ & $6 \%$ & $163 \mathrm{pe} / \mathrm{MeV}$ & 1.77 & 20 & $26 \%$ \\
\hline $\mathrm{DC}$ & $39010^{\prime \prime}$ & $16 \%$ & $200 \mathrm{pe} / \mathrm{MeV}$ & 0.81 & 8.2 & $7 \%$ \\
\hline RENO & $35410^{\prime \prime}$ & $15 \%$ & $230 \mathrm{pe} / \mathrm{MeV}$ & 1.0 & 16 & $14.5 \%$ \\
\hline
\end{tabular}



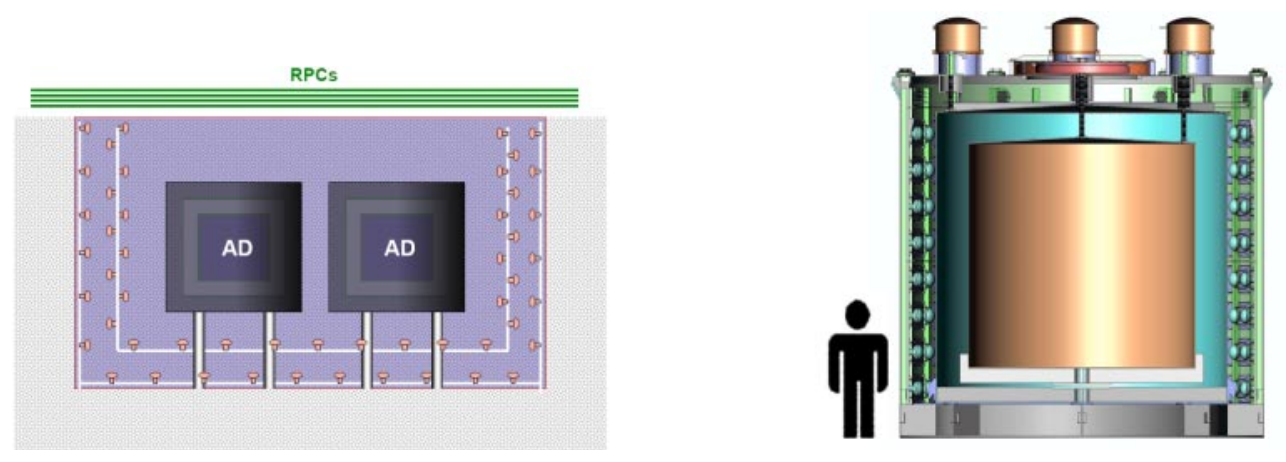

Fig. 3. - The detector arrangement (left panel) and detector module (right panel) of the Daya Bay experiment.

The Daya Bay experiment was initially proposed in 2003 while the construction started in 2007. The description of the experiment and detector systematic errors can be found in Ref. [20]. The civil construction completed at the end of 2010 and the detector installation completed in 2011. At the end of 2011, all the detectors in three experimental halls started to be operational and the first physics result was reported on March 8, 2012, using data from Dec. 24, 2011 to Feb. 17, 2012, a total of 55 days [17]. An updated result was reported later using 137 days of data, which confirmed non-zero $\theta_{13}$ at $7.7 \sigma[21]$. A factor of merit of the Daya Bay experiment is the possibility to cross check systematic errors by using two detector modules at the same site. Their observed numbers of neutrino events should be the same after known corrections. In fact, such a ratio is expected to be 0.981 . Our observation is $0.984 \pm 0.004$ (stat) \pm 0.002 (syst), in good agreement with the expectation. It proves that our systematic error is under control.

A new analysis using the measured energy spectrum of neutrinos was recently reported by the Daya Bay experiment [22]. In fact, the distorted energy spectrum of neutrinos can be an independent edivence of neutrino oscillation and $\Delta m_{e e}^{2}$ can be determined, in addition to $\sin ^{2} 2 \theta_{13}$. The difficulty is the non-linear response of the scintillator and readout electronics. By deploying several radioactive sources with well-defined gamma lines into the detector, the non-linearity curve of the detector response can be extracted with the help of background gamma lines and muon-produced ${ }^{12} \mathrm{~B}$ beta-decay spectrum, as shown in the left panel of Figure 4. Several validation models are built based on different parameterization and weighting of data constraints. They are consistent within $1.5 \%$. The prompt positron spectrum after the non-linearity correction at each site can be found in the right panel of Figure 4. The fitting results for both $\sin ^{2} 2 \theta_{13}$ and $\Delta m_{e e}^{2}$ are illustrated in the left panel of Figure 5, demonstrating the validity of the threeflavor oscillation model. The neutrino survival probability versus $L / E$ (the neutrino propagation distance over neutrino energy) in the right panel of Figure 5 shows that neutrinos are truly oscillating.

The Daya Bay experiment completed the summer maintenance and a comprehensive calibration program using both automatic and manual calibration system. The full detector started to be operational since Oct. 2012 and the data taking will continue for the next $3-5$ years. The precision of $\sin ^{2} 2 \theta_{13}$ is expected to reach $3-4 \%$ by the end of the experiment. 

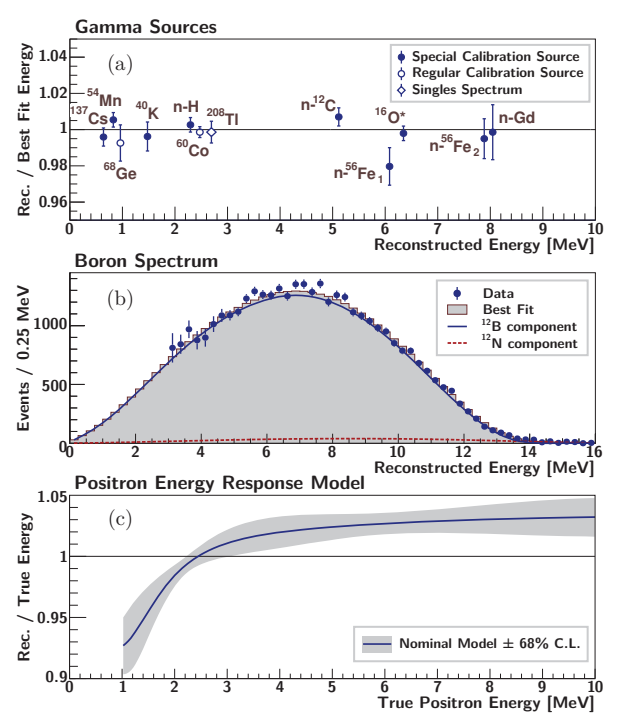

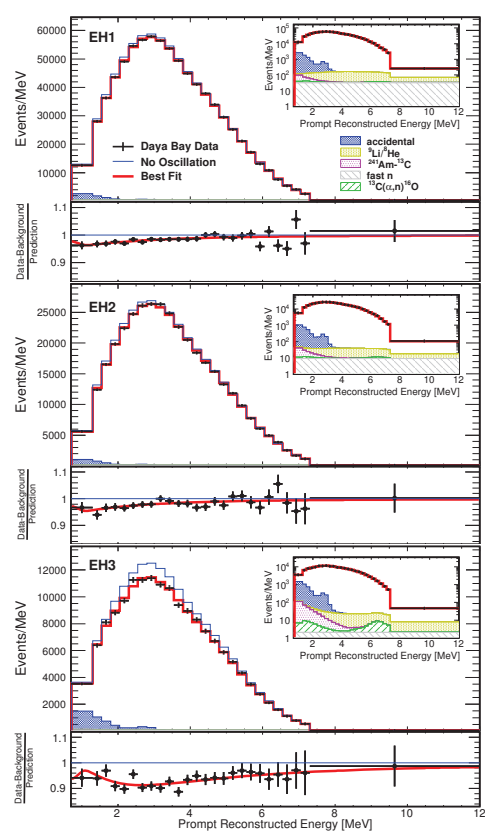

Fig. 4. - The non-linearity correction function (left panel) and prompt positron spectrum (right panel) of the Daya Bay experiment [22].

The RENO experiment in South Korea started data taking with both near and far detectors on Aug. 1, 2011. The first result was reported in April, 2012 by using 220 days of data, with a statistical significance of $4.9 \sigma$ [18]. An updated result with 403 days of data was reported in March, 2013 at the Neu-Tel workshop [23]. They measured the neutrino oscillation amplitude as $\sin ^{2} 2 \theta_{13}=0.100 \pm 0.010$ (stat) \pm 0.015 (syst). The energy spectrum and the ratio of near to far spectrum are shown in Figure 6 . The RENO experiment plans to take data for the next 5 years and the expected precision to $\sin ^{2} 2 \theta_{13}$ is $7 \%$.

The Double Chooz experiment started the far detector data taking at the beginning of 2011 and the near detector will be completed in 2014. They reported a first hint of non-zero $\theta_{13}$ at the end of 2011, and later various updates [24-26]. Without the near detector, Double Chooz experiment used several different methods, taking advantages that a single reactor may turn-off completely, so backgrounds can be measured precisely. By using combinations of rate plus shape analysis, the reactor rate modulation analysis, the Gd capture and Hydrogen capture analysis, the Double Chooz experiment obtained consistent results of $\sin ^{2} 2 \theta_{13}$, all at a statistical significance of about $3 \sigma$ level [24-26]. Once the near detector will be operational in 2014 , the final precision of $\sin ^{2} 2 \theta_{13}$ can reach $10 \%$.

It is clear that a combined fit of $\sin ^{2} 2 \theta_{13}$ from three reactor experiments is desired by the community. But a simple average is not adequate since some uncertainties are correlated and some are estimated by a different standard. The three reactor experiments agreed to work together to produce an appropriate average so that a best value of $\sin ^{2} 2 \theta_{13}$ can be available. 

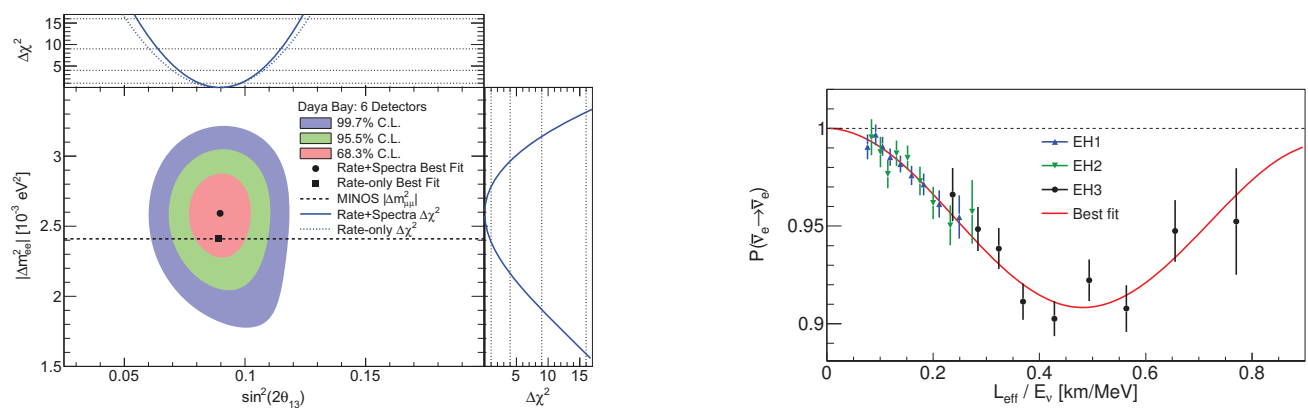

Fig. 5. - The allowed region (left panel) and neutrino survival probability versus $L / E$ (right panel) for the Daya Bay rates and spectra measurement [22].

\section{4. - Future experiments}

Reactor neutrino experiments will not stop here. A next generation experiment, called JUNO, is now under the planning stage in China [27]. The idea of the experiment is to place a detector at the oscillation maximum of $\theta_{12}$, where we are not only very sensitive to $\Delta m_{21}^{2}$ and $\theta_{12}$, but also sensitive to the interference between $\Delta m_{32}^{2}$ and $\Delta m_{31}^{2}$, hence to the neutrino mass hierarchy [28].

By using the following nominal values with a target mass of $20 \mathrm{kton}$, the energy resolution of $3 \% / \sqrt{E}(\mathrm{MeV})$, the total thermal power of $36 \mathrm{GW}$, the baseline of $53 \mathrm{~km}$ and a $1 \%$ uncertainty of $\Delta m_{\mu \mu}^{2}$, we can obtain the sensitivity to the mass hierarchy as shown in the left panel of Figure 7 [29]. In fact, there will be 60 reactor neutrino events per day in this detector. The backgrounds are mainly from random coincidences, at a level of about a few percent, and cosmic-ray-related processes, which amount to less than $1 \%$.

This experiment, at the oscillation maximum of $\theta_{12}$, can also measure precisely three

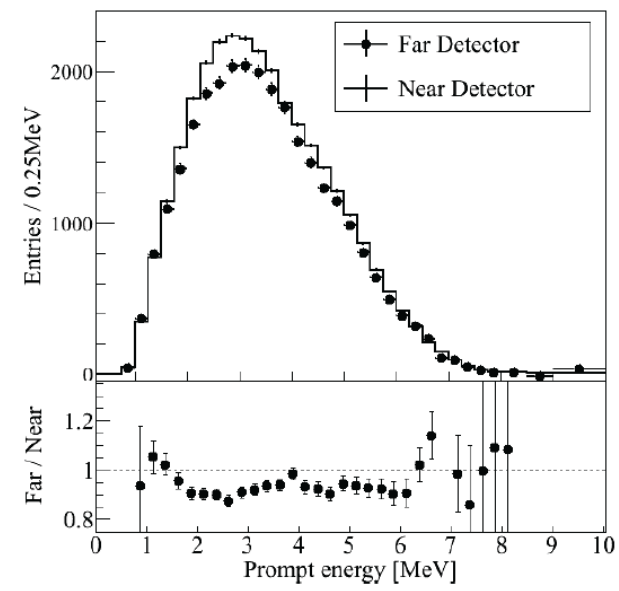

Fig. 6. - Results from the RENO experiment [23]: Energy spectrum of reactor neutrinos at near and far detectors, and their ratios. 

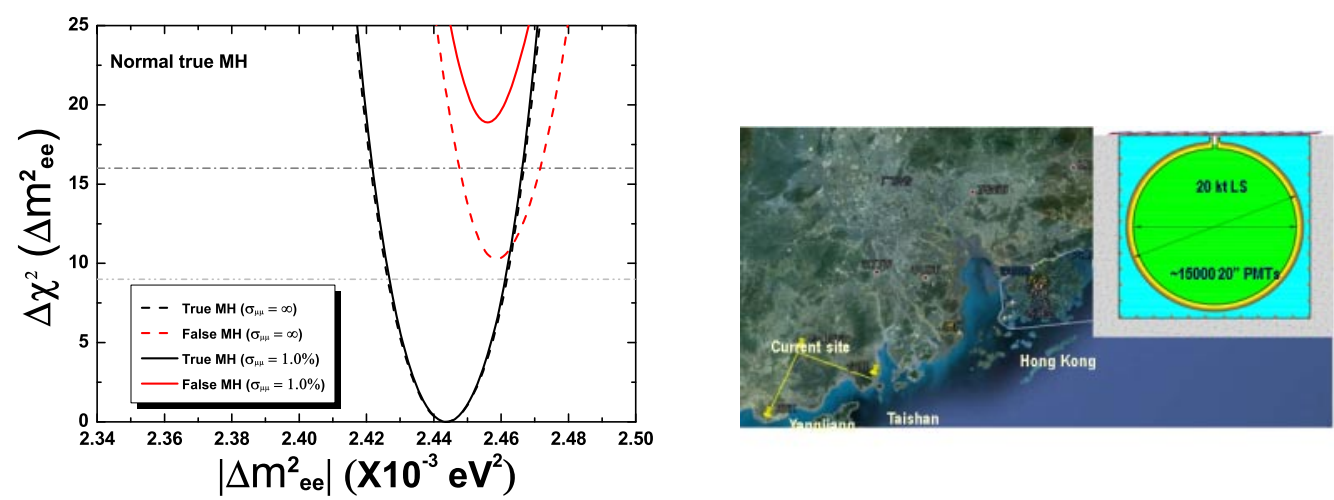

Fig. 7. - The mass hierarchy sensitivity (left panel) and arrangement (right panel) of the JUNO experiment.

of all the six oscillation parameters. Table III shows its capability. In fact, such a precision will be better than that of the CKM matrix, and the unitarity of the neutrino mixing matrix can be tested at a precision better than $1 \%$.

The scientific capabilities of the JUNO experiment can actually expand to astrophysics, covering supernova neutrinos, geo-neutrinos, solar neutrinos, and sterile neutrinos, etc.

The conceptual design of the detector is shown in the right panel of Figure 7. A large acrylic central detector with a diameter of 34.5 meters can house 20 kt liquid scintillator. A 37.5 meter diameter stainless steel tank can house all the phototubes and maintain a buffer region to shield radioactive backgrounds from the steel and phototube glass. In order to have a maximum energy resolution, the whole surface is covered with $20^{\prime \prime}$ phototubes, counting a total number of 15,000 . The whole detector is merged in a water pool to shield backgrounds and stabilize the working conditions. Water will be used as a Cerenkov detector to veto cosmic-rays while a tracking detector mounted at the top of the water pool is to track cosmic-rays to study backgrounds.

The experiment is located at a site with an equal distance to two reactor complexes. One is called Taishan which will house 4 reactor cores with a total thermal power of 18.4 GW. Two of them are now under construction. Another one is called Yangjiang which will have 6 reactor cores with a total thermal power of $17.4 \mathrm{GW}$. Four of them are now

TABLE III. - Precision of neutrino mixing parameters at present and in the future.

\begin{tabular}{|c|c|c|}
\hline & Current level & JUNO sensitivity \\
\hline$\Delta m_{21}^{2}$ & $3 \%$ & $0.6 \%$ \\
\hline$\Delta m_{31}^{2}$ & $5 \%$ & $0.6 \%$ \\
\hline $\sin ^{2} \theta_{12}$ & $6 \%$ & $0.7 \%$ \\
\hline $\sin ^{2} \theta_{23}$ & $6 \%$ & N/A \\
\hline $\sin ^{2} \theta_{13}$ & $10 \% \rightarrow \mathbf{4 \%}$ & $15 \%$ \\
\hline
\end{tabular}


under construction. The JUNO experiment is approved by the Chinese government for R\&D and the funding is available for the next two years. A detailed geological survey and the detailed civil design are now on-going. We expect to complete the preparation work up to 2014 and to start the civil construction in 2015. An international collaboration will be established soon and new comers are welcome. The experiment is expected to start operation in 2020.

A similar proposal by the RENO group, called RENO-50, is also under planning now.

It has been pointed out a few years ago that reactor neutrino fluxes may have been under-estimated so that the measured reactor neutrino flux may have a deficit, indicating a possible reactor neutrino oscillation with sterile neutrinos [30]. Subsequently, a number of new calculations confirmed the calculation while some argued that the significance of the deficit is not sufficient [31]. Indications of the oscillation with sterile neutrinos are also claimed by other experiments but global fits show that they are not the same oscillation [32]. Clearly there is a need to have more experimental evidence. Proposals include short baseline $(<100 \mathrm{~m})$ reactor experiments, accelerator experiments, and very strong radioactive source experiments [33].

\section{5. - Summary}

After the discovery of the neutrino oscillation, we now finally know all the neutrino mixing angles. Thanks to the large $\theta_{13}$, we can plan next generation experiments. In a few years from now, we will know all of the mixing angles and mass differences precisely. The unknown parameter, mass hierarchy, will be known in about 10 years [29, 34-36], while the most important parameter, CP phase, will be probably known in about 10-20 years $[37,38]$. This is an exciting field and exciting moment.

\section{REFERENCES}

[1] Particle Data Group, J. Beringer et al., Phys. Rev. D 86, 010001 (2012).

[2] Z.Z. Xing, Int. J. Mod. Phys. A 19, 1 (2004).

[3] C. Bemporad et al., Rev. Mod. Phys. 74, 297 (2002).

[4] B. Pontecorvo, Report PD-205, Chalk River Laboratory (1946). See S. M. Bilenky, arXiv:1210.3065, for a historical review.

[5] K. Eguchi et al., KamLAND Coll., Phys. Rev. Lett. 90, 021802 (2003).

[6] Q.R. Ahmad et al., SNO Coll., Phys. Rev. Lett. 87, 071301 (2001); Phys. Rev. Lett. 89, 011301 (2002).

[7] Y. Suzuki, talk given at NeuTel 2013 and the proceedings.

[8] G. Bellini et al., Borexino Coll., Phys. Rev. Lett. 108, 051302 (2012).

[9] G. Ranucci, talk given at this conference.

[10] Y. Fukuda et al., SuperKamiokande Coll., Phys. Rev. Lett. 81, 1562 (1998).

[11] H. Sekeya et al., SuperKamiokande Coll., arXiv:1307.3686.

[12] A. McDonald, talk given at Neutrino 2012 and the proceedings.

[13] P. Adamson et al., Minos Coll., Phys. Rev. Lett. 110, 251801 (2013).

[14] A. Gando et al., KamLAND Coll., arXiv: 1303.4667.

[15] H.B. Li, et al., TEXONO Collaboration, Phys. Rev. Lett. 90, 131802 (2003).

[16] K. N. Abazajian, et al., arXiv:1204.5379.

[17] F.P. An et al., Daya Bay Coll., Phys. Rev. Lett. 108, 171803 (2012).

[18] J.K. Ahn et al., RENO coll., Phys. Rev. Lett. 108, 191802 (2012).

[19] Y. Abe et al., Double Chooz Coll., Phys. Rev. Lett. 108, 131801 (2012).

[20] F.P. An et al., Daya Bay Coll., Nucl. Intrum. Meth. A 685, 78 (2012).

[21] F.P. An et al., Daya Bay Coll., Chinese Physics C 37, 011001 (2013). 
[22] F.P. An et al., Daya Bay Coll., arXiv:1310.6732. See also S. Jetter, talk given at NuFACT 2013 and the proceedings.

[23] S. H. Seo, talk given at the NeuTel 2013 and the proceedings.

[24] Y. Abe et al., Double Chooz Coll., Phys. Rev. D 86, 052008 (2012).

[25] Y. Abe et al., Double Chooz Coll., Phys. Lett. B 723, 66 (2013).

[26] C. Buck, talk given at this conference and the proceedings.

[27] Y.F. Wang, talk given at NeuTel 2013 and the proceedings.

[28] L. Zhan, et al., Phys. Rev. D 78, 111103 (2008); Phys. Rev. D 79, 073007 (2009).

[29] Y.F. Li et al., Phys. Rev. D 88, 013008 (2013).

[30] G. Mention et al., Phys. Rev. D 83, 073006 (2011).

[31] T.A. Mueller et al., Phys. Rev. C 83, 054615 (2011); P. Huber, Phys. Rev. C 84, 024617 (2011); C. Zhang et al., arXiv: 1303.0900; A.C. Hayes et al., arXiv: 1309.4146.

[32] J. Kopp, talk given at NeuTEL 2013 and the proceedings.

[33] M. Cribier, talk given at NeuTEL 2013 and the proceedings.

[34] M. Wurm et al., LENA coll., arXiv:1104.5620.

[35] M.G. Aartsen et al., PINGU and IceCube coll., arXiv:1306.5846.

[36] S. Choubey, talk given at Neutrino 2012 and the proceedings.

[37] C. Walter, talk given at Neutel 2013 and the proceedings.

[38] R. Wendell, talk given at the open meeting of HyperK, 2012. 